\title{
An oxygen enrichment attachment for use with humidified air
}

\author{
P. JEBSON, J. D E W A R, a n d J. W H I T E \\ Department of Anaesthetics, Hallamshire Hospital, Sheffield and Department of Anaesthetics, \\ Royal Infirmary, Edinburgh
}

\begin{abstract}
Jebson, P., Dewar, J., and White, J. (1974). Thorax, 29, 371-376. An oxygen enrichment attachment for use with humidified air. An oxygen enrichment attachment is described which fulfils the basic requirements for intubated patients. Using values for tidal volume and inspiratory time found in the type of patients for whom the attachment is intended, a range of mean inspired oxygen concentration has been given for $2,4,6,8$, and $101 /$ min oxygen flow.
\end{abstract}

Much work has been done on the design and performance of facemasks for the enrichment of inspired air with oxygen, and this has led to the production of masks which permit controlled oxygen therapy. The choice of the particular mask used depends on the desired range of oxygen concentration. This paper describes a new device for the oxygen enrichment of humidified air for use by patients breathing via an endotracheal or tracheostomy tube.

For such patients humidified air is led from its source through tubing which is joined to the airway by some form of connection. The connection must be considered as an integral part of the humidification system and should fulfil the following important criteria:

(a) The patient must be able to expire without restriction.

(b) Any excess flow from the humidifier should vent to the atmosphere.

(c) If the humidifier flow fails for any reason the patient must be able to breathe freely from the atmosphere without significant addition to his dead space.

(d) The need for tracheal toilet must be anticipated.

(e) The parts of the system must be capable of sterilization or be disposable.

$(f)$ The connection must be provided with a flow from the humidifier greater, or at least equal to, the peak inspiratory flow rate of the patient. There are many models of humidifiers but the majority do not produce a flow rate which exceeds that of the patient's inspiration. This last criterion and the problems of fulfilling it have been recognized by several workers when designing high-flow humidifiers (Feldman and Munro, 1963), and entrainment with its diluting effect on humidity and oxygen has been described more fully elsewhere (Nakamura and Jebson, 1972).

Since high flows of oxygen-enriched air are difficult and uneconomical to produce from a humidifier, particularly in conditions where a high inspired oxygen concentration is desired, we have added oxygen at the airway by means of a connection designed to work in conjunction with a humidifier producing $50 \mathrm{1} / \mathrm{min}$ of air. To avoid confusion with other connections, this has been named an 'oxygen enrichment attachment' (OEA).

\section{THE ATTACHMENT}

The prototype was constructed from nylon and steel. Figure 1 shows this in cross-section. The expiratory port (F) allows excess flow to escape and permits tracheal toilet without disconnection. The air limb (A) takes the delivery hose from the humidifier while the patient limb $(P)$ is inserted into the tracheostomy or endotracheal tube. Oxygen is added at the oxygen limb or nipple (O).

To evaluate the performance of the OEA a twopart study was carried out. These were:

1. a clinical assessment carried out on patients in a ventilation unit;

2. a quantitative evaluation of oxygen enrichment where volunteers or simulated respiration with a pump model were used. 


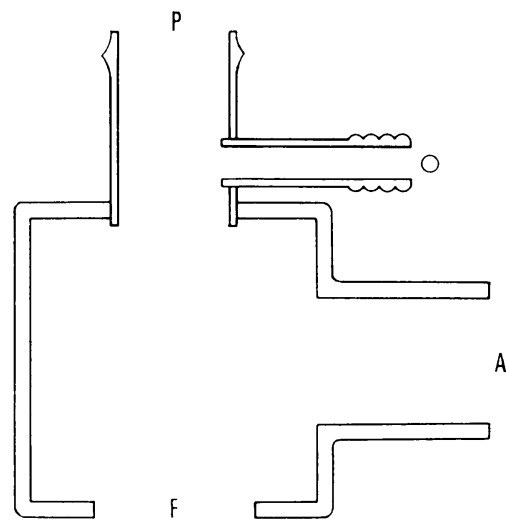

FIG. 1. Cross section of the oxygen enrichment attachment. A-air limb, F-expiratory port, $P$-patient limb, $O$-oxygen $\operatorname{limb}$.

\section{CLINICAL ASSESSMENT}

PATIENT STUdies The first object was to observe whether satisfactory arterial $\mathrm{Po}_{2}$ levels could be achieved in patients using the OEA with the air humidifier while oxygen was added to the oxygen limb of the attachment. Twenty-seven spontaneously ventilating patients with an endotracheal tube or tracheostomy were studied. The oxygen flow, fed to the oxygen nipple of the attachment, was selected as that flow which produced satisfactory oxygenation.

In a further seven patients, $0,2,4,6,8$, and $10 \mathrm{l} / \mathrm{min}$ were added to the oxygen nipple and the rise in arterial Po. was correlated to the oxygen flow.

The third object in the clinical assessment concerned the handling characteristics of the OEA as experienced by the patients, nursing, and medical staff.

\section{QUANTITATIVE EVALUATION}

END-TIDAL CONCENTRATION OF OXYGEN IN VOLUNTEERS A mouthpiece was fixed to the patient limb of the OEA and eight volunteers were asked to breathe through the attachment with the nose clipped; $50 \mathrm{l} / \mathrm{min}$ of air were continuously blown to the patient limb and the sampling lead of a mass spectrometer (model AEIMS4) was inserted into the mouthpiece. The oxygen profile of each respiration was recorded and the end-tidal oxygen was taken from this record.

No attempt was made to achieve set respiratory patterns provided the volunteers had settled down to a constant pattern. Five oxygen flows of between 2 and $10 \mathrm{l} / \mathrm{min}$ were evaluated individually.

Each oxygen flow rate was maintained for some time before actual measurements were made and re-equilibration with room air was allowed before proceeding to a higher oxygen flow rate. Frequent checks on the calibration of the spectrometer were carried out to ensure constant accuracy.

SIMULATED RESPIRATION WITH AN EXPERIMENTAL MODEL In order to study the behaviour of the OEA in more detail and predict inspired oxygen concentration for clinical usage, a Harvard ventilation pump ( $\mathrm{Pp})$ was used as a substitute for patients' lungs. The experimental model is shown (Fig. 2). The OEA was separated from the pump by a $150 \mathrm{ml}$ dead space (DS) containing mixing baffles. Air (A) and oxygen (O) were fed to the appropriate limbs of the attachment. To monitor oxygen concentration the sampling lead of the MS4 spectrometer was placed as shown in the diagram. The Harvard pump has a range of respiratory frequencies between 0 and 40 respirations per minute. This pump, however, produces a continuous sine wave form of respiration, i.e., no pause occurs between each respiratory cycle. This absence of a pause was anticipated and the effects of pause/no pause were studied as described below. Though a sine wave cannot be regarded as reproducing the truly normal wave form of respiration it was considered for the study as an acceptable substitute and has previously been used in an experimental mode (Flenley, Hutchison, and Donald, 1963).

The inspiratory and peak expiratory flow rates achieved by the pump at different frequencies were measured by a pneumotachograph and were found to lie within the physiological range for the respective stroke or tidal volume.

The oxygen profile obtained at sampling point (S) shows the inverse relationship of oxygen

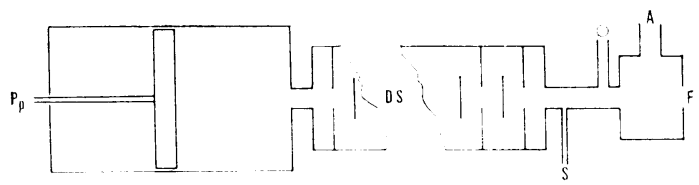

FIG. 2. The pump experimental model. A-air inlet, $O$-oxygen inlet, $S$-sampling lead to spectrometer, $D S$-dead space, F-expiratory port, Pp-Harvard ventilation pump. 
concentration to flow rate (Fig. 3). The first peak in oxygen concentration (1) corresponds with the low initial inspiratory flow rate. Oxygen concentration falls as the flow rate increases but, toward the end of inspiration as the flow rate again falls to lower levels, a second peak (2) in oxygen concentration appears. On expiration the oxygen concentration falls from peak 2 to an end-tidal plateau (3). The value of the plateau is not affected by the second peak (2) in inspired oxygen concentration because this peak remains in the dead space and does not enter the pump which may be considered to represent the alveoli. The value of the end-tidal oxygen concentration (3) is the effective mean inspired oxygen concentration in terms of alveolar ventilation and in the rest of this paper is referred to as 'mean inspired oxygen concentration level' $\left(\overline{\mathrm{F}}_{\mathrm{IO}} \mathrm{O}_{2}\right) \overline{\mathrm{F}}_{\mathrm{IO}}{ }_{2}$ was measured using the above system in the following series of experiments:

Effect of a pause between 'respirations' The pause was introduced by dropping alternate pump cycles and the effect on $\overline{\mathrm{F}}_{1 \mathrm{O}_{2}}$ was observed throughout the range frequencies and tidal volumes given below.

Effect of frequency and tidal volume Oxygen at $2,4,6,8$, and $10 \mathrm{l} / \mathrm{min}$ was fed to the attachment while frequency was varied between 5 and $40 \mathrm{cycles} / \mathrm{min}$ and stroke volume from 200 to $600 \mathrm{ml}$. Over 40 combinations of frequency and volume were thus used for each oxygen flow. The duration of inspiration and peak inspiratory flow
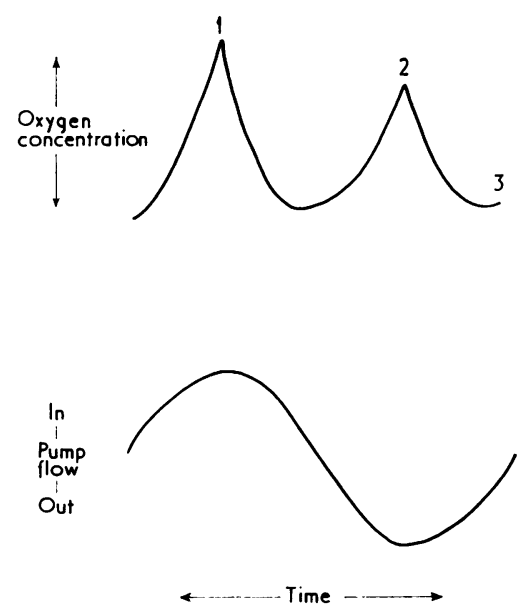

FIG. 3. Oxygen profile obtained with experimental model (diagrammatic). rate of all these combinations had previously been determined using a pneumotachograph.

Extent of air entrainment The flow of room air $(501 / \mathrm{min}$ ) to the OEA (which in the experiments simulated humidifier flow) was in turn increased, decreased, and completely stopped. Under these conditions $\overline{\mathrm{F}}_{\mathrm{IO}} \mathrm{z}_{2}$ was recorded using a range of pump settings as above.

\section{RESULTS}

PATIENT STUDIES Satisfactory oxygenation was achieved in the 27 patients who initially used the OEA.

The levels of $\mathrm{PaO}_{2}$ achieved in the seven patients for whom incremental flows of oxygen were fed to the attachment are shown in Table I. The amount of oxygen required to raise the $\mathrm{PaO}_{2}$ to an 'acceptable' level varied. In some patients an 'overshoot' occurred but it was established that $\mathrm{PaO}_{2}$ could be raised by this method. The only important criticism of the attachment was that it was rather heavy. This could be overcome by using a lighter plastic in construction. Cleaning and sterilization were easily carried out.

T A B L E I

CLINICAL ASSESSMENT OF OEA

\begin{tabular}{c|c|c|c|c|c|c}
\hline \multirow{2}{*}{ Patient } & \multicolumn{6}{c}{$\mathrm{PaO}_{2}$ on Air or with Added Oxygen (torr) } \\
\cline { 2 - 7 } & Air & $21 / \mathrm{min}$ & $41 / \mathrm{min}$ & $61 / \mathrm{min}$ & $81 / \mathrm{min}$ & $101 / \mathrm{min}$ \\
\hline 1 & 60 & 87 & 105 & 136 & 184 & - \\
2 & 67 & 93 & 121 & 178 & 194 & 198 \\
3 & 48 & 71 & $\overline{79}$ & 142 & 167 & -114 \\
4 & 50 & 64 & 79 & 84 & 100 & 114 \\
5 & 52 & 64 & 71 & 103 & -184 & 190 \\
6 & 69 & 96 & 125 & 176 & 184 & 175 \\
7 & 57 & 84 & 110 & 158 & 171 & \\
\hline
\end{tabular}

- No sample or result available.

QUANTITATIVE ASSESSMENT OF OXYGEN ENRICHMENT End-tidal concentration of oxygen Figure 4 shows end-tidal oxygen concentrations in eight volunteers who were breathing through the OEA while incremental amounts of oxygen were being added to the attachment. Each point represents the mean end-tidal $\mathrm{O}_{2}( \pm \mathrm{SE})$ of the eight volunteers at the appropriate flow of oxygen through the side limb. The linear behaviour of oxygen enrichment by the OEA and the levels of alveolar oxygen that can be achieved are apparent. It is also evident that a linear rise in inspired oxygen is produced where the added oxygen flow is increased in a similar manner. 


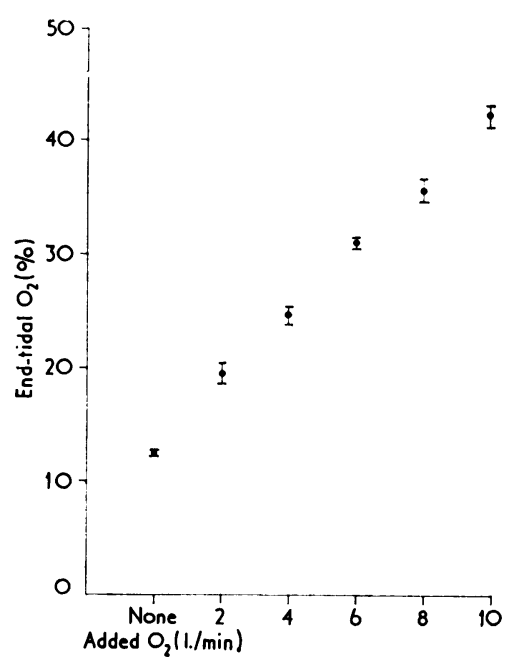

FIG. 4. End-tidal $O_{2}(\%)$ achieved in volunteers using the $O E A$.

Simulated respiration with the pump The $\overline{\mathrm{F}}_{1 \mathrm{O}_{2}}$ found with (1) the continuous form of respiration and (2) the cycle interrupted by a pause are compared in Table II. These results were obtained for frequencies of 10 and 20 cycles $/ \mathrm{min}$ and stroke volume $500 \mathrm{ml}$. The comparison made in Table II may be taken as characteristic of the attachment behaviour throughout the range of frequency and volume used, i.e., a pause did not significantly alter performance. This was confirmed by measuring mouth levels of oxygen in the volunteers during respiration. Oxygen enrichment by the attachment occurs only on inspiration and depends on the characteristics of inspiration.

Forty combinations of frequency and stroke volume were carried out to assess the effect, for each oxygen flow rate, of inspiratory patterns.

The important inspiratory characteristics of each breath were 'tidal volume' (stroke volume of the pump) and mean inspiratory flow rate. The

T A B L E I I

EFFECT ON MEAN INSPIRED $\mathrm{O}_{3}$ CONCENTRATION (\%) OF \begin{tabular}{c|c|c}
\multicolumn{2}{c}{ A PAUSE BETWEEN RESPIRATIONS } \\
\hline \multirow{2}{*}{$\begin{array}{c}\text { Added Oxygen } \\
(1 / \text { min) }\end{array}$} & \multicolumn{2}{|c}{ Mean Inspired $\mathbf{O}_{2}$ Conc. ( $\mathbf{F}_{2}$ ) } \\
\cline { 2 - 3 } & Continuous ( $\mathrm{f}=20 / \mathrm{min})$ & Pause ( $=10 / \mathrm{min})$ \\
\hline 2 & 29 & 28 \\
4 & 32 & 32 \\
6 & 39 & 40 \\
8 & 48 & 50 \\
10 & 57 & 55 \\
\hline
\end{tabular} latter for any given tidal volume depended on the duration of inspiration. Stroke volume and duration of inspiration have been used as the parameters of inspiration to be related to oxygen enrichment in the results.

Figure 5 demonstrates the relationship between $\bar{F} \mathrm{O}_{2}$ and duration of inspiration ('tidal volume' fixed at $500 \mathrm{ml}$ ). Figure 6 shows the relationship between $\overline{\mathrm{F}}_{\mathrm{IO}}$ and tidal volume (duration of inspiration fixed at $1 \mathrm{sec}$ ).

Effect of altering humidifier flow The manoeuvres of increased, decreased or abolished 'humidified' airflow $(501 / \mathrm{min})$ to the attachment had no effect on $\bar{F}_{10}$, at all settings of the pump.

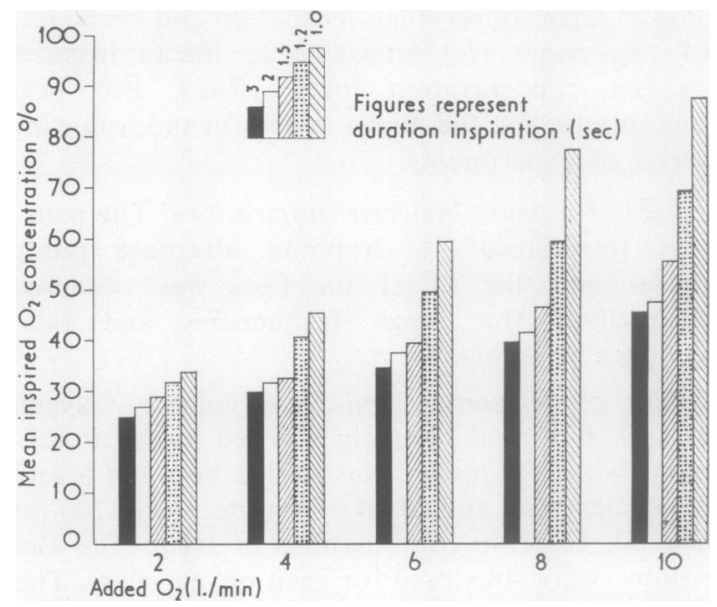

FIG. 5. 'Tidal volume' (pump stroke volume) $500 \mathrm{ml}$; effect of inspiratory time on inspired oxygen.

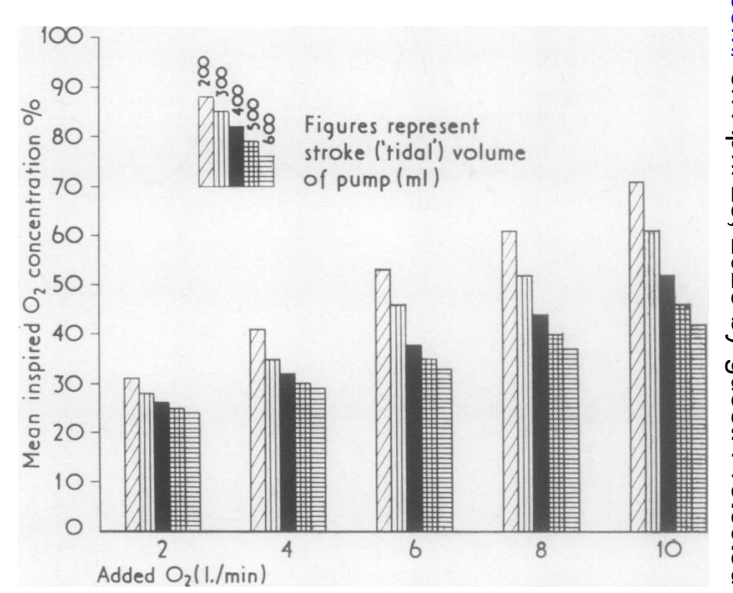

FIG. 6. Duration of inspiration 1 sec; effect of 'tidal volume' on inspired oxygen. 
It was apparent that where the flow was decreased or abolished ambient air was entrained during inspiration through the expiratory port $(F)$.

To confirm the relationship between inspiratory flow and air entrainment a stream of argon was placed just outside the expiratory port and the inspired argon concentration monitored in the patient limb. Argon was detected in the inspired gases when the inspiratory flow rate of the pump exceeded the flow rate of air from the humidifier.

\section{DISCUSSION}

As previously stated, much investigation has been carried out on the design and performance of facemasks for oxygen enrichment of inspired air in non-intubated patients (Flenley et al., 1963; Campbell and Gebbie, 1966).

Less attention has been paid to oxygen enrichment in orally and nasally intubated patients or patients who have undergone tracheostomy. To the problem of enrichment in such patients must be added the need for suitable humidification of the inspired gases as described in the introduction. The present study has assessed a new attachment for use with a humidified air system which fulfills the requirements outlined in the introduction. The dilution of the gases from the humidifier which occurs when an insufficient flow to cover inspiration is delivered to ' $T$ '-pieces of the type suggested by Bendixen et al. (1965) has already been made apparent (Nakamura and Jebson, 1972). In an effort to raise inspired oxygen levels a combination of the Gilston ' $T$ '-piece (Gilston, 1961) and the oxygen Venturi of the Venturimask has been described by Stoddart (1970). This Ventitube, as it has been called, uses the $28 \%$ or $35 \%$ Venturi tube from the respective Venturimasks as the delivery tube for a flow of oxygen containing water vapour or nebulized water particles in air. Though suitably low and predictable oxygen concentrations were described by this author over a range of tidal volumes and frequencies, only partial humidification occurred because of dilution of the wet oxygen by room air. To produce sufficient nebulized particles with the Ventitube, the rather high flow rate of $15 \mathrm{l} / \mathrm{min}$ of oxygen is required. Stoddart also evaluated the Gilston ' $T$ '-piece and observed that a considerable variation in oxygen concentration was found with this device.

Before the capacity of the OEA to fulfil clinical needs is discussed it is pertinent to describe the clinical conditions under which oxygen therapy in 'intubated patients' is required. First, there is the group of patients whose respiratory drive may be found to depend on hypoxia. Tracheostomy or intubation may be carried out to facilitate tracheobronchial toilet. This type of patient requires humidified inspired air with accurately controlled low levels of oxygen enrichment (25$30 \%$ inspired oxygen). Second, there is a larger group of patients who require greater amounts of oxygen enrichment. For example, the patient with a 'crushed chest' who, in the interval between coming off the ventilator and having his tracheostomy closed, requires humidified inspired air with varying oxygen enrichment (30-60\% inspired oxygen).

The OEA can fulfil the requirements of both groups of patients, but to predict as accurately as possible the inspired oxygen concentration for the patient requires definition of the range of tidal volume and duration of inspiration found in clinical practice.

Inevitably some respiratory variation is found from patient to patient and also in the individual patient, as described by Leigh (1970). After monitoring respiration in intubated 'respiratory' patients with the pneumotachograph, the authors regard $300-400 \mathrm{ml}$ as the usual range of tidal volume while the duration of inspiration varies from 1 to 1.2 seconds. These are lower than some 'normal' values quoted. From these data, a range of mean inspired oxygen concentrations has been predicted for each flow rate of oxygen added to the attachment (Table III). The range widens at higher flows of oxygen but this is relatively unimportant when high inspired oxygen levels are required in clinical practice. Larger tidal volumes can be expected to produce lower values for inspired oxygen than those predicted, providing the duration of inspiration remains within 1 to 1.2 seconds. Smaller tidal volumes produce the reverse effect. For respiratory values lying midway between 300 and $400 \mathrm{ml}$ and 1 and $1.2 \mathrm{sec}$, $21 / \mathrm{min}$ of oxygen added to the attachment will ensure a mean inspired oxygen concentration of up to $28 \% ; 41 / \mathrm{min}$ will increase this concentration

\section{T A B L E I I I}

PREDICTED RANGE OF INSPIRED $O_{2}$ CONCENTRATION FOR PATIENTS VT 300-400: INSPIRATORY TIME 1.0-1.2 SEC

\begin{tabular}{c|c}
$\begin{array}{c}\text { Oxygen added to } \\
\text { Attachment } \\
\text { (1/min) }\end{array}$ & $\begin{array}{c}\text { Range of Inspired } \\
\text { Oxygen Concentration } \\
\%\end{array}$ \\
\hline 2 & $26-30$ \\
4 & $32-40$ \\
6 & $37-50$ \\
8 & $44-58$ \\
10 & $52-66$ \\
\hline
\end{tabular}


to $35 \%$ and $61 / \mathrm{min}$ to just over $40 \% ; 81 / \mathrm{min}$ of additional oxygen will produce a mean inspired oxygen range near $50 \%$ while $101 / \mathrm{min}$ produces a maximum inspired oxygen up to $60 \%$. Higher levels of oxygen enrichment can be achieved by increasing the oxygen beyond the limit of $10 \mathrm{l} / \mathrm{min}$ used in this study.

\section{REFERENCES}

Bendixen, H. H., Egbert, L. D., Hedley-Whyte, J., Laver, M. B., and Pontoppidan, H. (1965). Respiratory Care, p. 113. Mosby, St. Louis.

Campbell, E. J. M. and Gebbie, T. (1966). Masks and tent for providing controlled oxygen concentration. Lancet, 1, 468.

Chamney, A. R. (1969). Humidification requirements and techniques. Anaesthesia, $24,602$.

Feldman, S. A. and Munro, J. A. (1963). A new blower-humidifier. British Medical Journal, $2,612$.

Flenley, D. C., Hutchison, D. C. S., and Donald, K. W. (1963). Behaviour of apparatus for oxygen administration. British Medical Journal, 2, 1081.

Gilston, A. (1961). A tracheostomy T-piece. Lancet, 1, 1326.

Leigh, J. M. (1970). Oxygen therapy at ambient pressure. In Scientific Foundations of Anaesthesia, edited by C. Scurr and S. Feldman. p. 205. Heinemann, London.

Nakamura, Y. and Jebson, P. (1972). Inspired oxygen concentrations using a humidifier/tracheostomy T-piece system. British Journal of Anaesthesia, 44, 61 .
Stoddart, J. C. (1970). Ventitube for providing oxygenenriched humidified air. Lancet, 2, 1166.

\section{ADDENDUM}

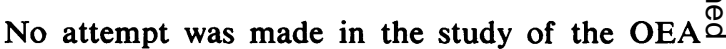
to evaluate the efficiency of the air blower type of humidifier with which the attachment is $\vec{\circ}$ intended for use.

Despite the recommendations of Chamney $\vec{\omega}$ (1969) little improvement has been made in the ability of such humidifiers to deliver warm $\times$ humidified air to the patient's airway. Certainly a $\subseteq$ larger surface of heated water is required for $\omega$ $50 \mathrm{l} / \mathrm{min}$ air flow than is at present provided, and counter measures should be introduced to avoid condensation in the delivery tube. It may be added that a filter put on the air intake of one $\vec{z}$ model appreciably decreases the flow from the humidifier. Although this will not affect oxygen enrichment by the OEA it will lead to entrain- ment of dry air through the expiratory port. The oxygen inspired from the attachment should be humidified and this could be carried out using the existing hot water bath of the humidifier. On preliminary experiment such a system appears to be satisfactory.

Requests for reprints to: Dr. P. Jebson, Department of Anaesthetics, University of Sheffield Medical School, Beech Hill Road, Sheffield S10 2RX. 